\title{
Avaliação numérica da influência da geometria do ferramental na geração de tensões residuais durante o processo de trefilação de barras de aço
}

\author{
Numerical evaluation of the die geometry \\ influence on the residual stresses \\ produced during the wire drawing \\ process of steel bars
}

Tiago Brun Coser ${ }^{1}$,
Tomaz Fantin de Souza $^{2}$, Alexandre da Silva Rocha

\author{
${ }^{1}$ Laboratório de Metalurgia Física - LAMEF - PPGE3M/UFRGS, Av. Bento Gonçalves, 9500, Porto Alegre, RS \\ e-mail: tiago.brun@ufrgs.com.br \\ ${ }^{2}$ Laboratório de Transformação Mecânica - LdTM - PPGE3M/ UFRGS, Av. Bento Gonçalves, 9500 Porto Alegre, RS \\ e-mail: tomazfs@yahoo.com.br; alexandre.rocha@ufrgs.br
}

\section{RESUMO}

Trefilação é um processo de fabricação que consiste na redução da área de seção transversal de um material, quando este atravessa uma matriz cônica, chamada fieira. Devido às características inerentes ao processo, as deformações são heterogêneas ao longo da seção do material, originando tensões residuais, que podem contribuir com o aparecimento de distorções, propagação de trincas e consequentemente acarretar em redução da vida útil do produto final. Neste trabalho, foi analisada a influência da geometria da fieira no perfil de tensões residuais geradas em um caso típico de trefilação de fio máquina para obter-se barras retas de aço AISI 1045, com redução em área de seção transversal de aproximadamente $11 \%$ e diâmetro inicial de 21,46 mm. O objetivo foi encontrar um design de ferramenta que reduza a magnitude das tensões residuais em relação a um design industrial típico com ângulo de trefilação de $15^{\circ}$ e único passe. Ensaios de compressão e do anel foram realizados para obter-se a curva de escoamento do material e o valor do coeficiente de atrito. Os modelos numéricos foram validados através da análise dos perfis das tensões residuais simuladas com as condições de equilíbrio impostas pelo problema, bem como com dados da literatura. Além disso, os valores das deformações principais e da pressão de contato (entre a barra e a fieira) simulados foram comparados com valores calculados. Inicialmente, foram simuladas condições com diferentes relações de região de calibração por diâmetro final, verificando-se que na faixa entre 0,4 a 0,6 ocorre uma diminuição nas tensões residuais axiais. Quando incluída uma região cônica dupla na fieira, foi encontrada uma grande redução nas tensões residuais axiais na superfície, de 65\% em relação ao design industrial, mostrando ser esta uma solução promissora para a melhoria do projeto de fieiras de trefilação de fio máquina.

Palavras-chave: trefilação, tensões residuais, simulação computacional.

\begin{abstract}
Wire drawing is a metalworking process used to reduce the cross-section of a wire by pulling the wire through drawing dies. The generated strains are non-homogeneous through the cross section of the material, leading to residual stresses that can contribute to the appearance of shape distortions, crack propagation and shortening of the product life time. In this work, the influence of the die geometry in the generated residual stresses on a typical case of wire rod drawing operation (production of straight AISI 1045 steel bars with $11 \%$ reduction from an initial diameter of $21,46 \mathrm{~mm}$ ) was investigated. The objective was to obtain a new tool design able to reduce the residual stresses in relation to a typical design of the tools which includes the use of a drawing angle of $15^{\circ}$ in one single pass. Compression and ring compression tests were performed in order to obtain the material stress-strain curve and the friction coefficient of the process, respectively. The developed numerical models were initially validated by analyzing simulated residual stress profiles regarding
\end{abstract}


the equilibrium conditions for the problem, as well as by comparison with literature data. Furthermore, the principal strains and the contact pressure (between the bar and the die) values obtained from the simulation were compared with calculated ones. Initially, simulations were carried out for different bearing lengths and it was found that for relations of bearing length to diameter of the drawn bar between 0.4 and 0.6 , a reduction in the axial residual stresses can be achieved. A large reduction in the axial residual stresses at the surface of $65 \%$ in relation to the typical industrial design was found in the case of the design with a double conical region, showing to be a promising solution for die design improvement.

Keywords: wire drawing, residual stresses, numerical simulation.

\section{INTRODUÇÃO}

Barras de aço são massivamente aplicadas na indústria automotiva como, por exemplo, na fabricação de eixos para transmissão de potência ou de amortecedores, dentre outras utilizações. Estas barras são usualmente fabricadas através do processo de trefilação, um processo de conformação mecânica, que consiste em reduzir a área de seção transversal de uma barra, fazendo-a passar através de uma matriz cônica, chamada fieira, por meio de uma força de tração aplicada na saída da matriz [1,2]. A deformação plástica é obtida a partir da combinação de tensões trativas e compressivas, que são desenvolvidas através da força de trefilação e da configuração geométrica da fieira [2]. Dentre as vantagens deste processo, pode-se citar o excelente acabamento superficial, a ótima precisão dimensional e o ganho de resistência mecânica ao final deste processo [3].

Entretanto, um fato inerente ao processo de trefilação, é a ocorrência de deformações heterogêneas em toda a seção da peça, causando o aparecimento de tensões residuais no produto final [1]. Atualmente, pode-se dizer que o comportamento do perfil de tensões residuais após a trefilação, para reduções maiores que 1\%, é conhecido. Durante a última década, diversos autores estudaram tanto experimental, quanto analiticamente, através da utilização de simulações numéricas computacionais, os perfis das tensões residuais ao final processo $[1,4,5,6,7,8,9,10]$. A Figura 1 ilustra os perfis de tensões residuais esperados para as três direções principais. Para a direção axial, as tensões superficiais são trativas e tendem a compressivas conforme se analisa em profundidades maiores. Essas tensões, por exemplo, estão relacionadas com o aparecimento de empenamentos e distorções durante processos de corte, retífica e tratamento térmico, podendo gerar perda de matéria-prima durante o processo de manufatura [7,11]. Já para a direção radial, a tensão deve ser nula na superfície por questões de equilíbrio, sendo totalmente compressiva ao longo da seção. Por fim, para a direção tangencial, tensões trativas aparecem na superfície e compressivas no núcleo, onde os valores de tensões residuais tangenciais e radiais devem ser iguais, devido à geometria da barra. As tensões tangenciais são uma das causas mais comuns de fratura de devido à ocorrência de trincas longitudinais no material. Essas trincas se estendem da superfície do material em direção à linha de centro, e podem chegar até mesmo a alguns metros de comprimento na direção axial. As tensões residuais superficiais na direção tangencial são a principal força motriz para o crescimento desse tipo de trincas [12].

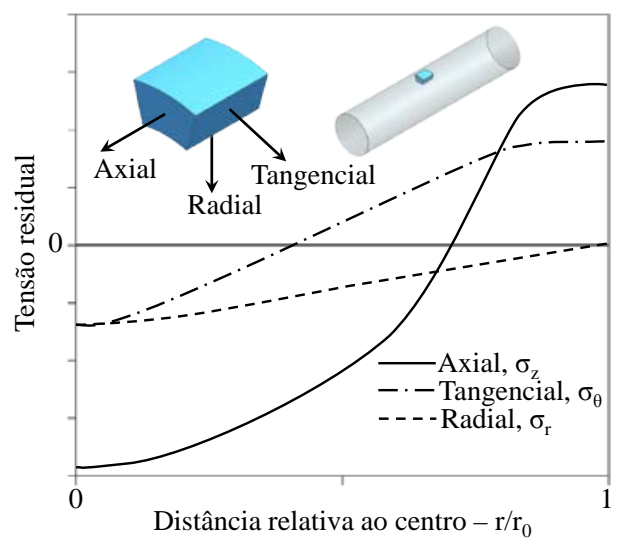

Figura 1: Perfil de tensões residuais esquemático para as três direções principais.

Diferentes parâmetros do processo de trefilação como: geometria da ferramenta, coeficiente de atrito, grau de redução e velocidade de trefilação tem uma influência significativa nas propriedades finais do material [11]. Dessa forma, o conhecimento de fatores que alteram as propriedades dos produtos semiacabados durante a fabricação é de fundamental importância e se torna o primeiro passo para a melhoria do produto 
final [8].

Além disso, a partir da década de 60, o uso de análises numéricas utilizando, dentre outros, o método dos elementos finitos, tem permitido aperfeiçoar estudos de diferentes processos de fabricação de maneira que se evitem erros de projeto, diminuição do número de try-outs e gastos desnecessários com materiais e processos adicionais [13,14]. Portanto, a simulação computacional se torna uma ferramenta bastante interessante para avaliar as tensões residuais presentes em uma barra trefilada e avaliar as modificações que podem ser feitas no processo para otimizar os perfis destas tensões residuais. Há a necessidade do desenvolvimento de métodos capazes de controlar e reduzir a magnitude das tensões. Uma alternativa eficaz é a alteração dos parâmetros da fieira, que podem levar a um design otimizado para uma maior homogeneidade das deformações plásticas [11,12,15].

KUBOKI et al. [6] verificou, que a partir da adição de uma pequena redução na última etapa do processo de trefilação, as tensões residuais podem ser reduzidas consideravelmente. Esta redução compensa o alongamento diferente entre a região superficial e o núcleo da barra. Inicialmente, alguns trabalhos foram escritos propondo a adição um último estágio de trefilação destinado à realização da redução mínima, chamado de skin pass [6]. Entretanto, do ponto de vista prático, isso significa a adição de um processo, levando a um aumento do número de fieiras e passes, significando um aumento dos custos de produção. Dessa forma, alguns autores propuseram a modificação da geometria da fieira de forma a introduzir essa pequena redução final, em vez de adicionar uma nova fieira ao final do processo [11,15].

Neste trabalho, o processo de trefilação de barras de AISI 1045 foi simulado numericamente propondo-se alterações na geometria da fieira. A influência de diferentes parâmetros nas tensões residuais geradas foi avaliada. Inicialmente, diferentes comprimentos da região de calibração foram propostos e analisados. Além disso, duas alterações em relação à geometria convencional foram estudadas - introdução de uma região cônica dupla e introdução de uma saliência na região de calibração - a fim de se obter uma redução final mínima, responsável pela redução das tensões residuais. Os resultados obtidos foram comparados entre si bem como com os da geometria convencional, sendo possível determinar qual o melhor design para o caso em estudo considerando o perfil de tensões residuais gerados.

\section{MATERIAIS E MÉTODOS}

Foi escolhido um processo industrial típico de trefilação de barras de aço AISI 1045 com uma redução de diâmetro de 21,46 mm para 20,25 mm para a realização da modelagem. O processo foi simulado utilizando o software de elementos finitos ABAQUS ${ }^{\circledR}$. Um modelo numérico axissimétrico foi desenvolvido para descrever o processo.

Ensaios de compressão e do anel foram realizados para que fossem obtidos a curva de escoamento do material e o coeficiente de atrito do processo como dados de entrada no software. Foram verificadas, através de dados encontrados na literatura, as condições de equilíbrio e a geometria do problema, com o intuito de validar o modelo. Além disso, as deformações principais e pressão de contato obtidas a partir da simulação foram comparadas com valores obtidos a partir de equações conhecidas.

\subsection{Ensaio de compressão para obtenção da curva de escoamento do material}

Ensaios de compressão foram realizados para a obtenção da curva de escoamento. Optou-se por este ensaio devido à obtenção de maiores deformações que as obtidas em um ensaio de tração convencional [16]. Cinco corpos de prova cilíndricos com $10 \mathrm{~mm}$ de diâmetro e $15 \mathrm{~mm}$ de altura foram confeccionados a partir de uma barra de aço AISI 1045 não trefilada e ensaiados em uma prensa hidráulica com capacidade de 40 toneladas e velocidade de $6 \mathrm{~mm} / \mathrm{s}$. A partir das cincos curvas de escoamento obtidas, foi construída uma curva média que é mostrada na Figura 2. 


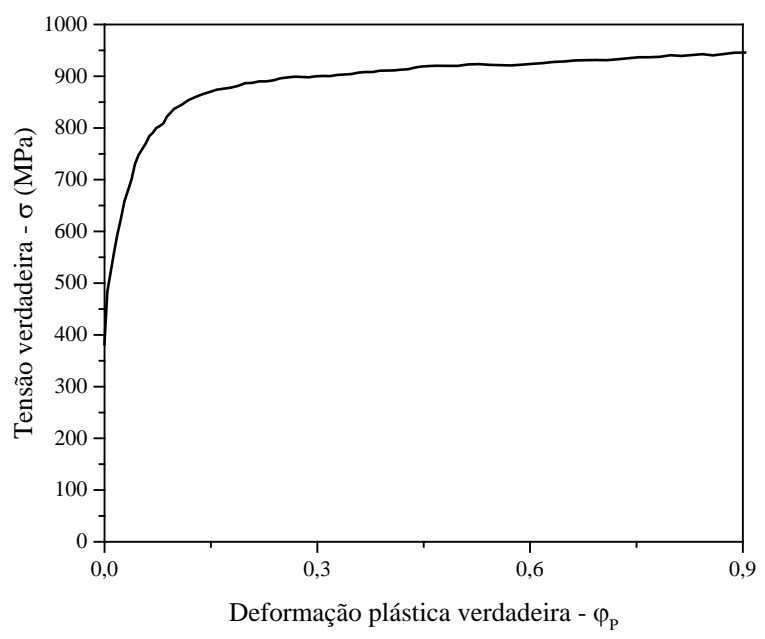

Figura 2: Curva de escoamento para o aço AISI 1045 obtida por ensaio de compressão.

A curva apresenta a tensão verdadeira $(\sigma)$ em função da deformação plástica verdadeira $\left(\varphi_{\mathrm{P}}\right)$, que pode ser facilmente determinada a partir da Equação 1

$$
\begin{aligned}
& \varphi_{\mathrm{T}}=\varphi_{\mathrm{P}}+\varphi_{\mathrm{E}} \\
& \varphi_{\mathrm{P}}=\varphi_{\mathrm{T}}-\frac{\sigma}{\mathrm{E}}
\end{aligned}
$$

Onde $\varphi_{\mathrm{T}}$ é a deformação total verdadeira, $\varphi_{\mathrm{E}}$ é a deformação elástica. $O$ valor do módulo de elasticidade (E) foi obtido por DAGNESE [17], sendo ele 206 GPa para o material em questão.

\subsection{Ensaio do anel para estimativa do coeficiente de atrito}

Um dos métodos mais tradicionais para se avaliar o atrito em processos de conformação mecânica é a realização do ensaio de compressão do anel. Nesse teste, um corpo de prova em forma de anel com faces planas é comprimido entre dois punções até uma redução conhecida.

O ensaio deve simular as condições de processo, assim, para o caso da trefilação, por exemplo, a região de contato do punção com a peça deve ser confeccionada com o mesmo material da fieira, bem como o anel deve ser feito a partir da barra trefilada. As condições de lubrificação também devem ser respeitadas [18].

Uma grande vantagem desse teste é que não é necessária a utilização de equipamentos para mensurar a força, por exemplo. Basta medir a redução de altura e variação do diâmetro interno. De posse desses valores, deve-se compará-los com curvas de calibração que mostram várias reduções em altura para diferentes valores de coeficiente de atrito de Coulomb $(\mu)$. Essas curvas podem ser obtidas por diversas análises, uma delas sendo por simulação computacional [18].

No presente trabalho, punções foram confeccionados a partir do material da fieira, WC-Co, e o mesmo tipo de lubrificante utilizado em aplicações industriais foram empregados na compressão do anel. As dimensões dos anéis foram de $20 \mathrm{~mm}$ para o diâmetro externo, $10 \mathrm{~mm}$ para o diâmetro interno e $6 \mathrm{~mm}$ de altura. Esses corpos de prova foram submetidos a reduções de, aproximadamente, 15, 30 e 45\%, sendo que dois ensaios foram realizados para cada redução, tomando-se o resultado como a média. Foi utilizada para o ensaio uma prensa manual com velocidade de, aproximadamente, $0,1 \mathrm{~mm} / \mathrm{s}$.

As curvas de calibração foram geradas através da simulação do ensaio de compressão do anel utilizando o software ABAQUS®. Um modelo axissimétrico com as mesmas dimensões dos corpos de prova foi desenvolvido, utilizando 1250 elementos do tipo CAX4R e variando-se os valores de coeficiente de atrito de $0,05,0,1,0,15$ e 0,2 .

A Figura 3 apresenta a sobreposição dos resultados experimentais e as curvas de calibração obtidas através da simulação computacional. Pode-se perceber que a curva obtida através do ensaio ficou próxima à curva de calibração para o valor de coeficiente de atrito de 0,1 . Dessa forma, este foi o valor adotando duran- 
te as simulações.

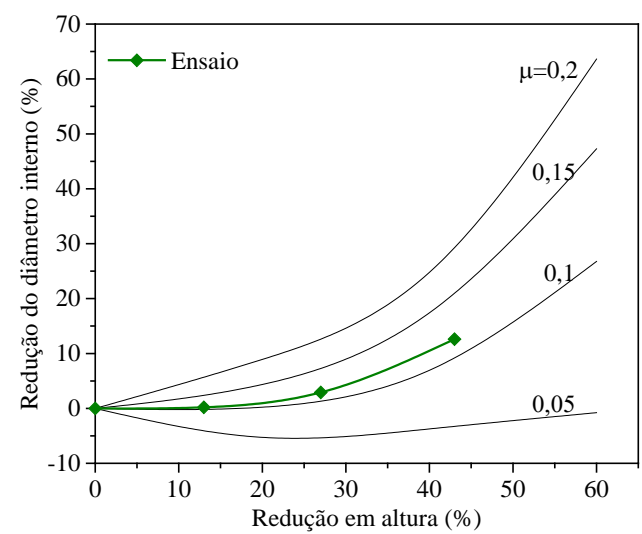

Figura 3: Curvas de calibração para o coeficiente de atrito variando de 0,05 a 0,2 e comparação com os resultados experimentais obtidos.

\subsection{Modelagem do processo de trefilação}

Um modelo axissimétrico para o processo, apresentado na Figura 4, foi proposto, devido à geometria do problema. Dessa forma, um menor tempo computacional pôde ser alcançado e uma maior faixa de parâmetros pôde ser avaliada. A trefilação foi simulada aplicando a velocidade de processo aos nós da extremidade da barra, forçando-a a passar pela ferramenta fixa. Uma geometria convencional de fieira foi considerada e definida como rígida. Além disso, não foram considerados efeitos térmicos acoplados. A Tabela 1 apresenta os demais parâmetros utilizados na simulação do processo.

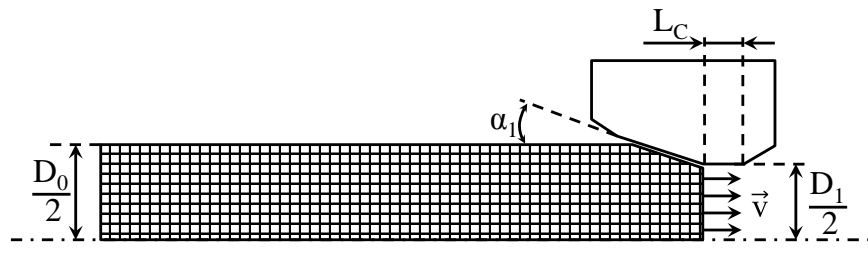

Figura 4: Modelo axissimétrico desenvolvido para simulação do processo de trefilação.

Tabela 1: Parâmetros utilizados na simulação do processo de trefilação

\begin{tabular}{l|l}
\hline Análise & Axissimétrica \\
\hline Elementos & $8400(21 \times$ x 400, CAX4R $)$ \\
\hline Tamanho médio do elemento & $0,5 \mathrm{~mm}$ \\
\hline Ângulo de fieira $\left(2 \alpha_{1}\right)$ & $15^{\circ}$ \\
\hline Diâmetro inicial $\left(\mathrm{D}_{0}\right)$ & $21,46 \mathrm{~mm}$ \\
\hline Diâmetro final $\left(\mathrm{D}_{1}\right)$ & $20,25 \mathrm{~mm}$ \\
\hline Velocidade de trefilação & $1250 \mathrm{~mm} / \mathrm{s}$ \\
\hline Coeficiente de atrito $(\mu)$ & 0,1 \\
\hline Material & AISI 1045 \\
\hline Módulo de elasticidade & $206 \mathrm{GPa}$ \\
\hline Coeficiente de Poisson & 0,3 \\
\hline
\end{tabular}

\subsection{Validação numérica}

A validação numérica foi realizada da seguinte forma: primeiramente, os perfis das tensões residuais obtidos através da simulação foram analisados e comparados com as condições de equilíbrio impostas pelo problema, bem como com dados obtidos na literatura. Por fim, realizou-se a comparação de valores para pressão de 
contato e para as deformações principais obtidos via simulação e cálculos analíticos.

Na Figura 5 são apresentados os perfis das tensões residuais para as três direções principais. O comportamento obtido é semelhante ao apresentado na Figura 1. Para a direção axial foram encontrados valores extremamente altos quando comparados com valores experimentais encontrados na literatura $[7,8]$. Este fenômeno já foi evidenciado por autores como ATIENZA et al. [4], DE SOUZA [5], KUBOKI et. Al. [6] e SOARES [10] que comparam resultados experimentais e numéricos utilizando diferentes softwares. A tensão residual radial tende a zero na superfície da barra e, na região central, o valor é igual ao da direção tangencial, satisfazendo as condições de equilíbrio.

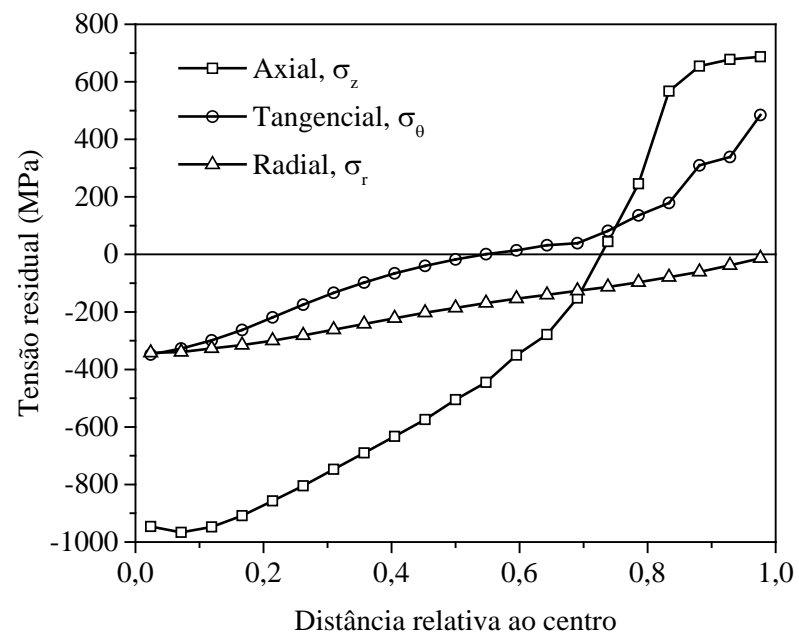

Figura 5: Perfis de tensões residuais obtidos via simulação para as três direções principais.

Subsequente à análise dos perfis de tensões, a pressão de contato entre a barra e a fieira foi avaliada. O valor obtido através da simulação foi comparado com a Equação 3, desenvolvida por JOHNSON e ROWE [19]:

$$
\mathrm{p}=\frac{\mathrm{F}}{\mathrm{S}\left(\mu \cos \alpha_{1}+\operatorname{sen} \alpha_{1}\right)}
$$

Onde: p é a pressão de contato, $\mathrm{F}$ é a força de trefilação e $\mathrm{S}$ é a área da superfície de contato. A força de trefilação foi calculada através de duas formulações devidas a Siebel e a Sachs [20], dadas pelas Equações 4 e 5 , respectivamente. Dessa forma, valores de pressão foram também calculados utilizados cada uma das fórmulas.

$$
\begin{aligned}
& \mathrm{F}=\mathrm{A}_{1} \varphi_{\mathrm{A}} \mathrm{k}_{\mathrm{fm}}\left(1+\frac{\mu}{\alpha_{1}}+\frac{2}{3} \frac{\alpha_{1}}{\varphi_{\mathrm{A}}}\right) \\
& \mathrm{F}=\mathrm{A}_{1} \mathrm{k}_{\mathrm{fm}}\left\{\left(1+\frac{1}{\mu \operatorname{cotg} \alpha_{1}}\right)\left[1-\left(\frac{\mathrm{A}_{1}}{\mathrm{~A}_{0}}\right)^{\mu \operatorname{cotg} \alpha_{1}}\right]+0,77 \alpha_{1}\right\}
\end{aligned}
$$

Onde: $A_{0}$ e $A_{1}$ correspondem à área inicial e final da seção transversal da barra respectivamente, $k_{f m}$ é a tensão de escoamento média para a redução e $\varphi_{A}$ é a deformação verdadeira em área $\left(\varphi_{A}=\ln \left(A_{1} / A_{0}\right)\right)$.

A Tabela 2 apresenta a comparação entre os valores obtidos pela simulação e o calculado. Os valores mostraram-se próximos, sendo que o valor obtido pela simulação foi 19,7\% superior em relação à fórmula de Siebel e 23,71\% em relação à fórmula de Sachs. As diferenças encontradas entre a simulação e as equações podem ser atribuídas ao fato de que o valor apresentado para a pressão simulada se refere à média de valores na região de contato, aumentando quando se aproxima da região de transição entre a região de trabalho e de calibração. Já as equações utilizadas consideram a pressão com sendo uniforme na superfície de contato, o que acaba sendo responsável por essa diferença. 
Tabela 2: Comparação entre os valores de pressão de contato simulados e calculados. Valores em MPa.

\begin{tabular}{l|l|l}
\hline SIMULAÇÃO & $\begin{array}{l}\text { EQUAÇÃO DE } \\
\text { SIEBEL }\end{array}$ & $\begin{array}{l}\text { EQUAÇÃO DE } \\
\text { SACHS }\end{array}$ \\
\hline 1288,2 & 1076,53 & 1041,3 \\
\hline
\end{tabular}

Além da avaliação da pressão de contato, foram comparados os valores calculados e simulados para a deformação plástica. A partir da lei da constância de volume pode-se chegar às seguintes formulações apresentadas pelas Equações 6,7,8.

$$
\begin{aligned}
& \varphi_{\mathrm{l}}=-\varphi_{\mathrm{A}}=-\ln \frac{\mathrm{A}_{1}}{\mathrm{~A}_{0}} \\
& \varphi_{\mathrm{r}}=\ln \frac{\mathrm{D}_{1}}{\mathrm{D}_{0}} \\
& \varphi_{\mathrm{t}}=\ln \frac{2 \pi \mathrm{D}_{1}}{2 \pi \mathrm{D}_{0}}=\ln \frac{\mathrm{D}_{1}}{\mathrm{D}_{0}}
\end{aligned}
$$

Onde $\varphi_{1}$ é a deformação verdadeira longitudinal, $\varphi_{\mathrm{r}}$ é a deformação verdadeira radial, $\varphi_{\mathrm{t}}$ é a deformação verdadeira tangencial (ou circunferencial) [20].

A Tabela 3 apresenta a comparação entre os valores simulados e calculados para as deformações principais. Os valores obtidos por simulação se referem à média da deformação de 6 elementos superficiais na região central da barra. Os resultados ficaram muito próximos, apresentando diferenças de 1,49\%, 1,88\% e 1,58\% para a deformação longitudinal, radial e tangencial, respectivamente. Através das três validações realizadas, foi possível afirmar, portanto, que o modelo proposto descrevia corretamente o processo e a análise da influência da geometria do ferramental na geração de tensões residuais pôde ser avaliada.

Tabela 3: Comparação entre os valores de deformação verdadeira obtidos via simulação e equações.

\begin{tabular}{l|l|l|l}
\hline & LONGITUDINAL & TANGENCIAL & RADIAL \\
\hline SIMULAÇÃO & 0,1181 & $-0,0591$ & $-0,0593$ \\
\hline EQUAÇÃO & 0,1164 & $-0,0582$ & $-0,0582$ \\
\hline
\end{tabular}

\subsection{Alterações na geometria da fieira}

Primeiramente, a influência do comprimento da região de calibração foi estudada e logo após, com base na literatura, alterações na geometria da fieira foram propostas, aplicando as modificações na fieira propostas por ASAKAWA et al. [11] e KUBOKI et al. [15].

A região de calibração não envolve redução adicional. Em geral, seu comprimento $\left(\mathrm{L}_{\mathrm{C}}\right.$, mostrado na Figura 4) é determinado em função do diâmetro final $\left(D_{1}\right)$ da barra trefilada, com o seu valor usual variando entre 30 e 50\% [9]. Seis condições foram simuladas, conforme a Tabela 2, e os seus respectivos perfis gerados foram comparados.

Tabela 4: Comprimentos da região de calibração simulados.

\begin{tabular}{c|c}
\hline COMPRIMENTO $(\mathbf{m m})$ & $\mathbf{L}_{\mathbf{c}} / \mathbf{D}_{\mathbf{1}}(\mathbf{\%})$ \\
\hline 0,5 & 4,94 \\
\hline 1 & 9,88 \\
\hline 2,5 & 24,69 \\
\hline 5 & 49,38 \\
\hline 7,5 & 74,07 \\
\hline 8,5 & 83,95 \\
\hline
\end{tabular}


ASAKAWA et al. [11] propôs a introdução de uma saliência dividindo a região de calibração. A Figura 6 a) ilustra a alteração proposta. A redução é feita até um diâmetro intermediário $\left(\mathrm{D}_{1 / 2}\right)$ muito próximo ao final $\left(D_{1}\right)$ e, então, na região de calibração é introduzida a saliência responsável pela redução mínima final. Essa protuberância pode ser definida a partir do ângulo $\theta$ e a diferença entre o diâmetro intermediário e o final, denominado $\Delta \mathrm{h}$.

Já no trabalho de KUBOKI et al. [15], foi proposta a introdução de uma segunda região cônica responsável pela redução mínima que, teoricamente, teria o mesmo efeito do processo skin pass. A Figura 6 b) ilustra esse novo conceito de fieira. Através da introdução da segunda região cônica define-se um diâmetro intermediário $\left(\mathrm{D}_{1 / 2}\right)$ muito próximo ao diâmetro final $\left(\mathrm{D}_{1}\right)$, gerando-se a redução mínima, com um ângulo $\left(2 \alpha_{2}\right)$ inferior ao de trabalho $\left(2 \alpha_{1}\right)$.

a)

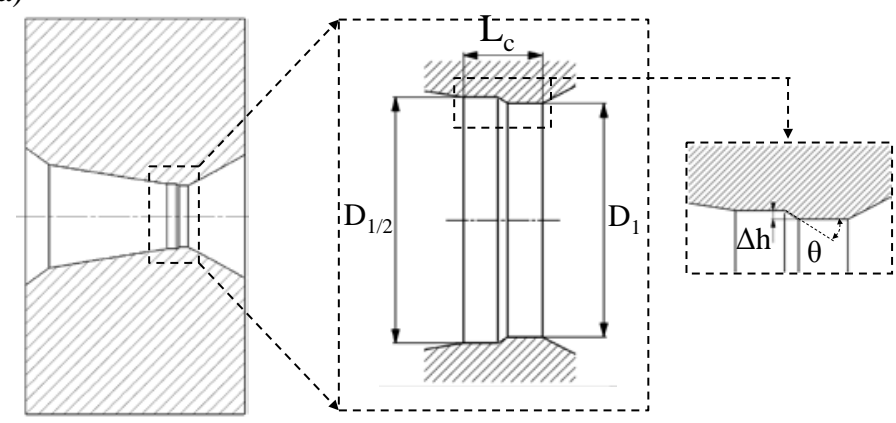

b)

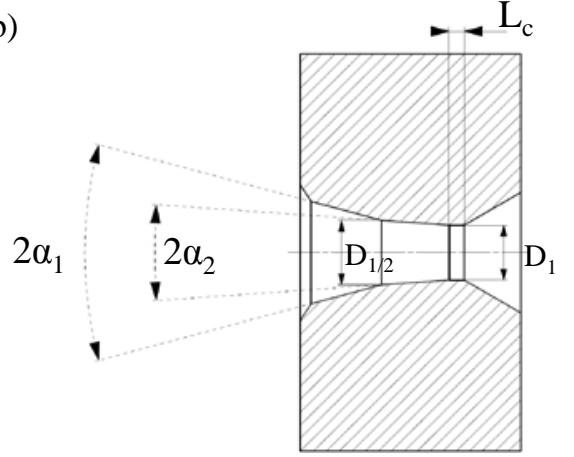

Figura 6: Alterações da geometria da fieira a) Fieira com protuberância proposta por ASAKAWA et al. [11] b) Fieira com região cônica dupla proposta por KUBOKI et al.[15].

A Tabela 5 mostra os parâmetros utilizados para as duas alterações da geometria da fieira encontradas na literatura para obter-se uma região de redução mínima. Além disso, também são mostrados os parâmetros utilizados para a configuração convencional da ferramenta.

Tabela 5: Parâmetros utilizados nas simulações propondo alterações na geometria da fieira.

\begin{tabular}{l|c|c|c}
\hline PARÂMETRO & $\begin{array}{l}\text { FIEIRA CON- } \\
\text { VENCIONAL }\end{array}$ & $\begin{array}{l}\text { FIEIRA COM } \\
\text { SALIÊNCIA }\end{array}$ & $\begin{array}{l}\text { FIEIRA COM } \\
\text { REGIÃo CôNICA } \\
\text { DUPLA }\end{array}$ \\
\hline $\mathrm{D}_{0}(\mathrm{~mm})$ & 21,463 & 21,463 & 21,463 \\
\hline $\mathrm{D}_{1 / 2}(\mathrm{~mm})$ & - & 20,46463 & 20,46463 \\
\hline $\mathrm{D}_{1}(\mathrm{~mm})$ & 20,25 & 20,25 & 20,25 \\
\hline Redução mínima & - & $1 \%$ & $1 \%$ \\
\hline $\begin{array}{l}\text { Comprimento da região de } \\
\text { calibração - L }(\mathrm{mm})\end{array}$ & 5 & 5 & 5 \\
\hline $\begin{array}{l}\text { Ângulos de trabalho da fieira }- \\
2 \alpha_{1}\end{array}$ & $15^{\circ}$ & $15^{\circ}$ & $15^{\circ}$ \\
\hline $\begin{array}{l}\text { Ângulo da segunda região cô- } \\
\text { nica - } 2 \alpha_{2}\end{array}$ & - & - & $1^{\circ}$ \\
\hline$\Delta \mathrm{h}(\mathrm{mm})$ & - & 0,21463 & - \\
\hline Ângulo da protuberância - $\theta$ & - & $15^{\circ}$ & - \\
\hline
\end{tabular}

\section{RESULTADOS E DISCUSSÃO}

\subsection{Influência do comprimento da região de calibração}

A Figura 7 apresenta os perfis de tensões residuais para a direção axial e tangencial, ambas apresentam valores trativos na superfície e isso pode causar dificuldades durante o processo de manufatura ou prejudicar a 
vida em fadiga do componente final. Verifica-se que há uma maior variação de resultados entre os modelos para as tensões residuais axiais (Figura 7 a) e, além disso, nessa direção é onde se verificam os maiores valores trativos na superfície. Entretanto, para a direção tangencial (Figura 7 b), a influência do comprimento da região de calibração é desprezível. Além disso, deve ser notado que a fieira e a barra de aço foram consideradas concêntricas sem haver uma inclinação entre as duas. É esperado que o comprimento da região de calibração tenha maior influência caso uma pequena inclinação da fieira em relação à barra seja considerada, representando melhor o caso real.
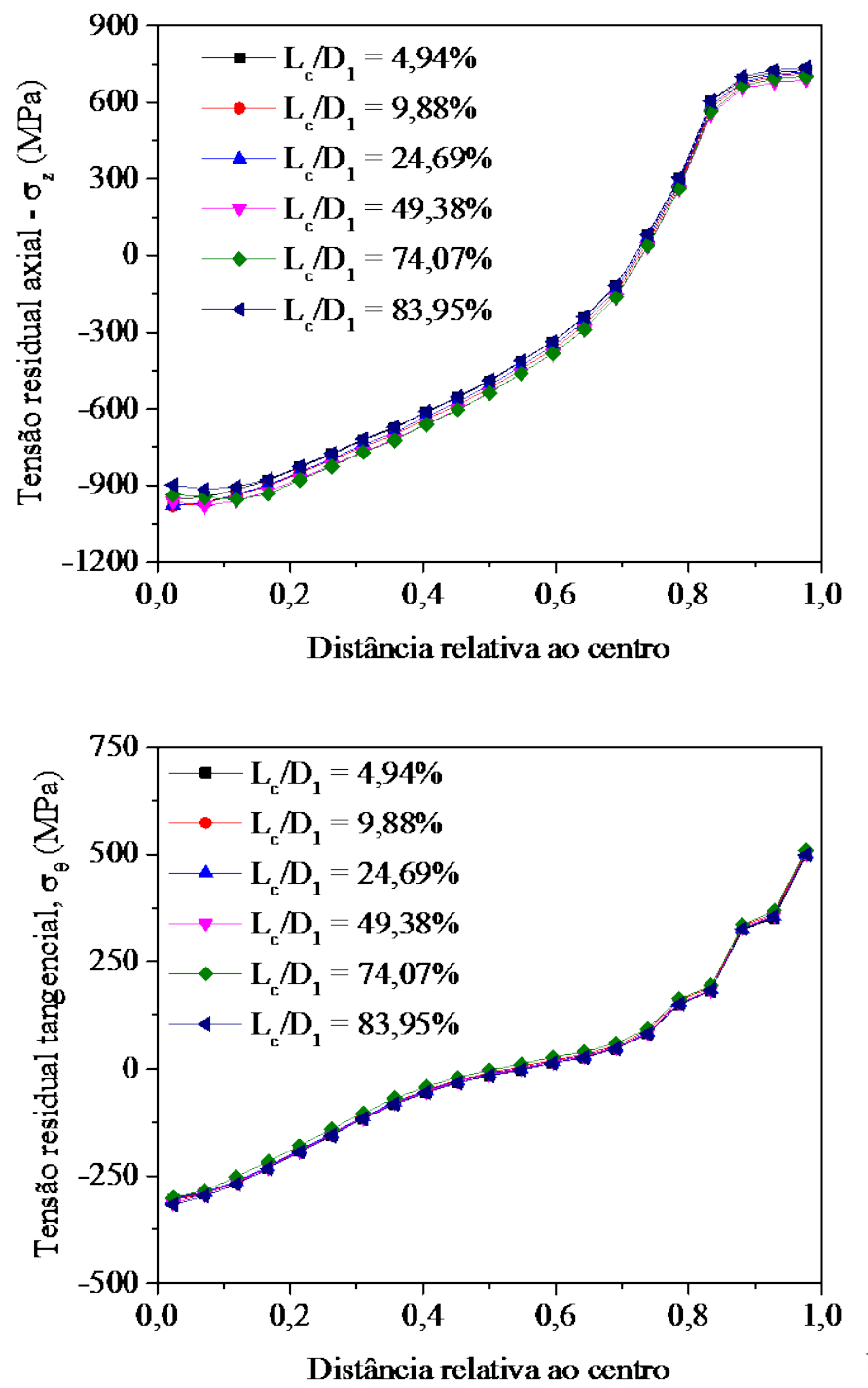

a)

b)

Figura 7: Perfis de tensões residuais para diferentes comprimentos da região de calibração, a) axial; b) tangencial.

A Figura 8 apresenta valores de tensão residual axial na superfície em relação aos diferentes comprimentos da região de calibração estudados. O maior valor de tensão residual axial obtido para esse caso foi $726 \mathrm{MPa}$, correspondente ao maior comprimento da região de calibração, enquanto o menor valor foi de 689 MPa, para o comprimento de $5 \mathrm{~mm}\left(\mathrm{~L}_{\mathrm{C}} / \mathrm{D}_{1}=49,38 \%\right)$, representado uma queda de 6,5\%.

Os resultados indicam que a relação $L_{C} / D_{1}$ na faixa entre 0,4 e 0,6 apresentam os menores valores de tensões residuais superficiais. ASAKAWA [11] sugere que para uma maior relação entre o comprimento de calibração e o diâmetro final do material, haverá redução no gradiente de tensões residuais. No presente trabalho, evidencia-se que para altíssimas relações, maiores que 0,6 , o valor das tensões residuais axiais superficiais volta a aumentar, demonstrando que existe uma faixa para o comprimento da região de calibração que permite reduzir o gradiente das tensões residuais. 


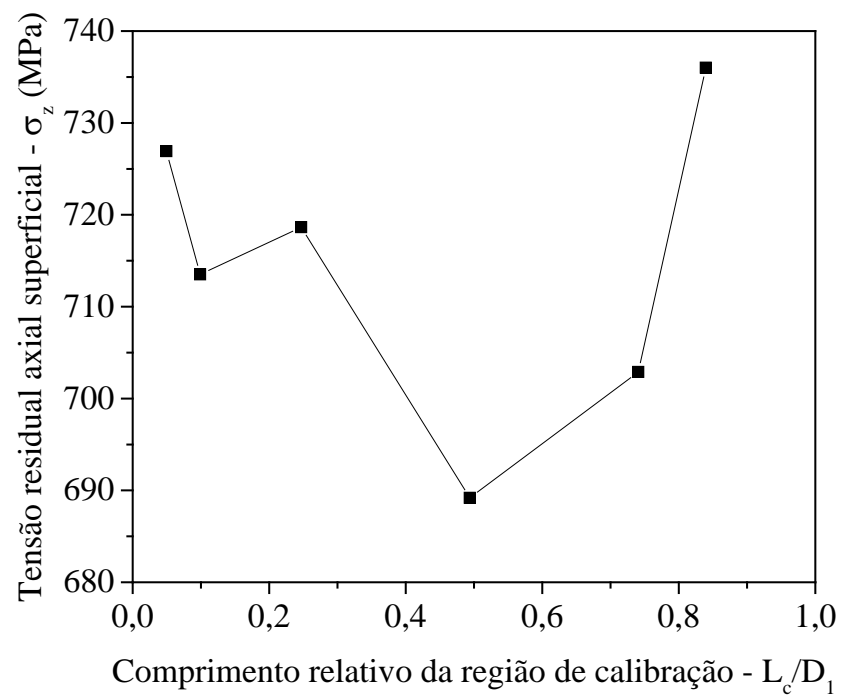

Figura 8: Relação entre o comprimento relativo da região de calibração $(\mathrm{Lc} / \mathrm{D} 1)$ e a tensão residual axial superficial $\left(\sigma_{z}\right)$.

\subsection{Introdução de uma redução final mínima visando diminuir tensões residuais}

A Figura 9 apresenta os perfis de tensões residuais para as três direções comparando a fieira convencional com as duas alterações propostas. Para a direção axial, as tensões residuais superficiais apresentaram valores de 235MPa para o modelo com região cônica dupla e 635 MPa com inserção de uma protuberância na região de calibração, indicando uma redução de $65 \%$ e $7 \%$ respectivamente, em relação à geometria convencional. Este resultado está de acordo com a literatura [11,15] e adicionalmente mostra uma comparação entre os diferentes designs para o presente caso estudado. Apesar de a literatura indicar que ambas as geometrias de fieira propostas por ASAKAWA et al. [11] e KUBOKI et al. [15] levariam a reduções das tensões residuais, não seria antes possível apontar qual o melhor método para ser aplicado no presente caso. Os resultados deste trabalho demonstram claramente que o uso da fieira com região cônica dupla apresenta um potencial muito maior para redução das tensões.

Já para a direção tangencial, as tensões superficiais apresentaram valores de 303 MPa e 278 MPa para os modelos com região cônica dupla e com protuberância, respectivamente representando uma redução de 39,4\% para o primeiro e 44,4\%, para o segundo caso. É possível perceber, que há uma redução considerável nas tensões residuais trativas das barras, tanto para a direção axial quanto para a tangencial, no centro e na superfície das barras.

Para a direção radial, é possível perceber, através da Figura 9 c), que o perfil das tensões residuais apresenta valores próximos a zero na superfície do material, como esperado, e valores compressivos em direção ao centro da barra, onde a fieira convencional apresentou um valor de $308 \mathrm{MPa}$, a fieira com protuberância, 292 MPa e a fieira com região cônica dupla, 225 MPa. A fieira com protuberância permitiu reduzir em $5 \%$ e a fieira com região cônica dupla em $27 \%$ os valores compressivos das tensões residuais para o centro da barra. 


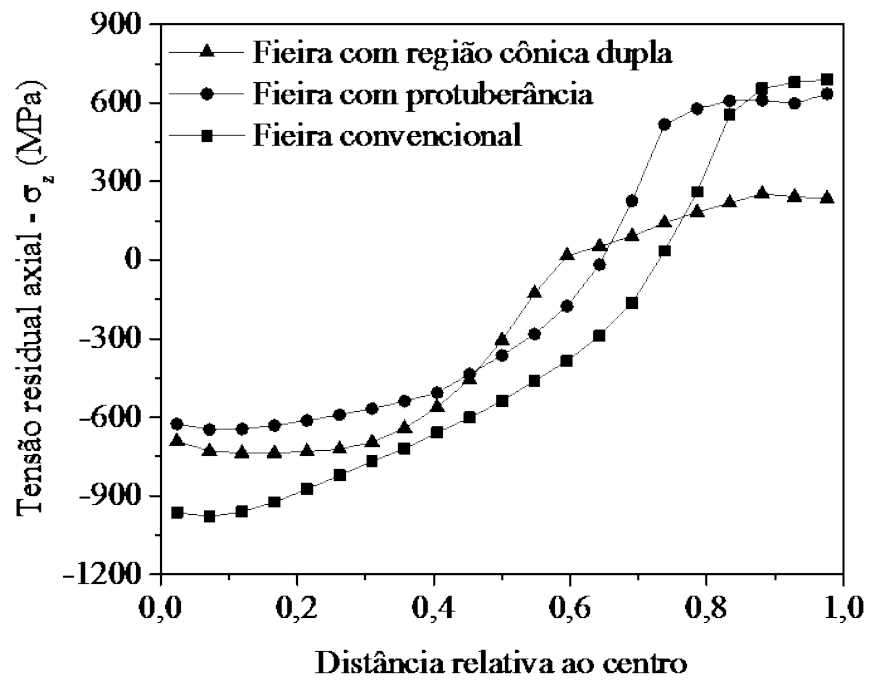

a)

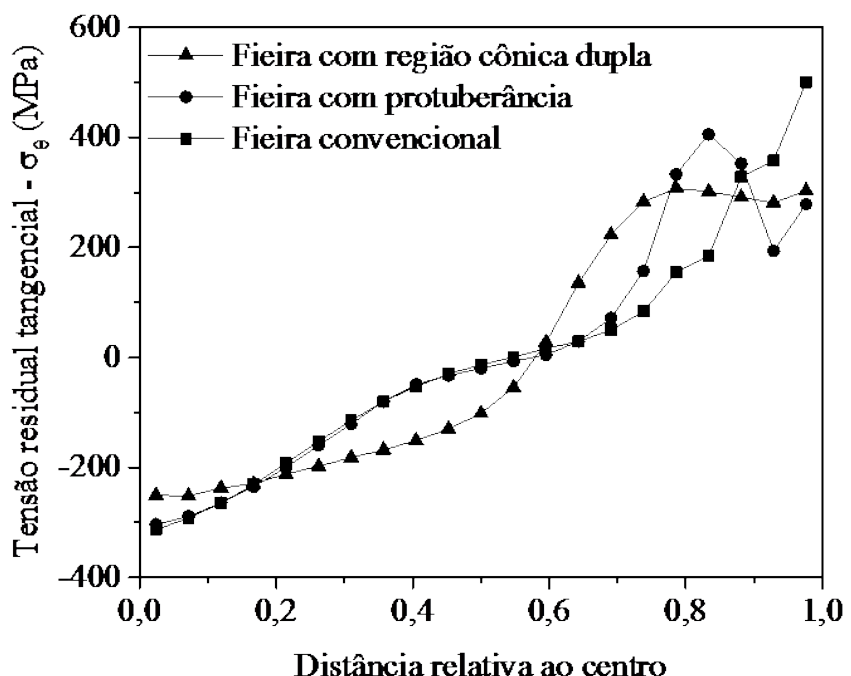

b)

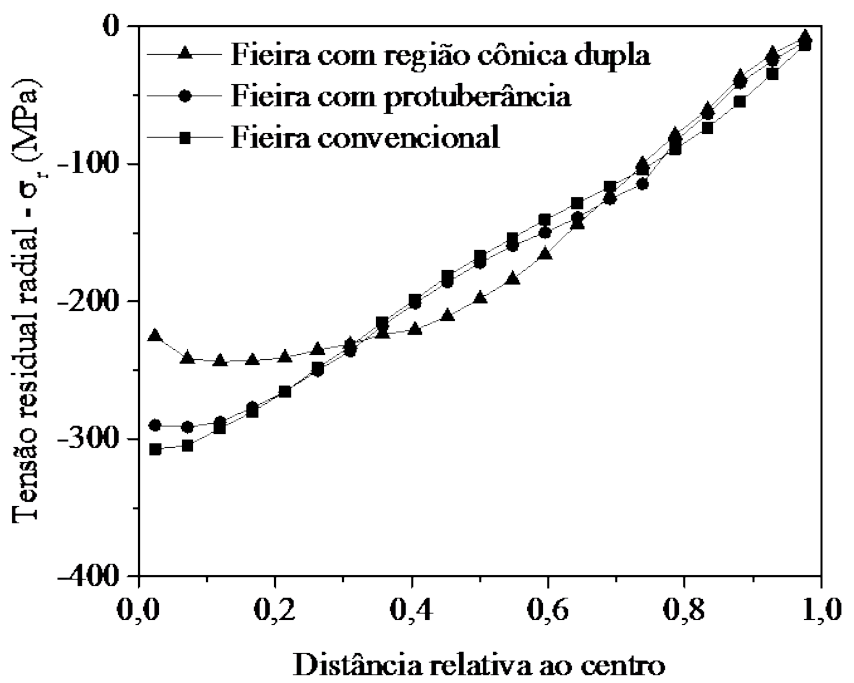

C)

Figura 9: Perfis de tensões residuais para as modificações propostas da fieira, a) axial; b) radial; c) tangencial.

Os resultados para a fieira com região cônica dupla são semelhantes aos obtidos por KUBOKI [15], que obteve uma maior redução das tensões para a direção axial, pois utilizou um segundo semiângulo $\left(\alpha_{2}\right)$ ainda menor que o proposto neste trabalho. Entretanto, do ponto de vista prático, é importante avaliar o au- 
mento da força de trefilação devido a um ângulo excessivamente pequeno, como afirmado pelo próprio autor em seu trabalho. No caso da fieira com protuberância, valores excessivamente pequenos de $\Delta \mathrm{h}$ podem ser difíceis de serem obtidos na manufatura da fieira, bem como uma análise do desgaste nessa região, devido à alta concentração de tensões, deve ser realizada.

Outro fato a ser notado é que, para as direções axial e radial, a geometria de ferramenta com região cônica dupla mostrou melhores resultados do que a fieira com protuberância. Já para a direção tangencial, na superfície do material, a fieira com protuberância apresenta resultados ligeiramente melhores que a outra geometria. Portanto, comparando-se os resultados entre as alterações propostas pode-se afirmar que, teoricamente, uma fieira com região cônica dupla apresentará um melhor perfil de tensões residuais em comparação com a fieira convencional e a com protuberância.

As diferenças encontradas para os resultados das tensões residuais devido à alteração na geometria da fieira devem-se ao fato de que a inserção de uma segunda redução mínima, logo após a redução principal permite uma deformação plástica menos heterogênea ao longo da área de seção transversal do material.

\section{CONCLUSÕES}

Uma análise numérica foi realizada para avaliar-se a influência de diferentes parâmetros sobre a geração de tensões residuais após o processo de trefilação. Duas alterações na fieira foram propostas com a finalidade de se introduzir uma redução final mínima. Uma diminuição de $65 \%$ na tensão residual axial superficial foi obtida com a introdução da região cônica dupla. Já para a direção tangencial, para a superfície do material, a fieira com protuberância na região de calibração apresenta resultados ligeiramente melhores que a fieira com região cônica dupla (44,4\% contra 39,4\% de redução).

Os resultados obtidos através deste trabalho confirmam os resultados relatados na literatura, de que a introdução de uma redução mínima na geometria da fieira após a deformação principal permite desenvolver deformações menos heterogêneas ao longo da área de seção transversal do material, o que ocasionará uma redução no perfil das tensões residuais. Adicionalmente, uma comparação direta foi feita entre dois métodos distintos para redução das tensões residuais, permitindo verificar que a utilização de um fieira com região cônica dupla mostra-se mais promissora do que a inserção de uma protuberânica na região de calibração. Além disso, foram simulados diferentes comprimentos da região de calibração, verificou-se, que para este parâmetro, a faixa de 0,4 a 0,6 da relação entre o comprimento desta região e o diâmetro final do material proporciona uma diminuição nas tensões residuais axiais na superfície da barra.

\section{AGRADECIMENTOS}

Agradecemos ao CNPq, CAPES e FINEP pelo apoio financeiro através do programa BRAGECRIM (Brazilian German Collaborative Research Initiative in Manufacturing Technology).

\section{BIBLIOGRAFIA}

[1] ATIENZA, J. M., Tensiones Residuales en Alambres de Aceros Trefilados, Tese de D.SC, E.T.S.I. de Caminos/Departamento de Ciencia de Materiales/Universidad Politécnica de Madrid, Madrid, Espanha, 2001.

[2] DIETER, G. E., KUHN, H.A., SEMIATIN, S.L., Handbook of Workability and Process Design, Materials Park - OH, ASM International, 2003.

[3] DIETER, G. E., Mechanical Metallurgy, 3. ed., New York, McGraw-Hill,1986.

[4] ATIENZA, J. M., RUIZ-HERVIAS, J., MARTINEZ-PEREZ, M.L., et al., "Residual stresses in cold drawn pearlitic rods”, Scripta Materialia, v. 52, n. 12, pp. 1223-1228, Jun. 2005.

[5] SOUZA, T. F., Simulações Computacionais para a Análise e Minimização das Tensões Residuais no Processo de Trefilação, PPGE3M/UFRGS, Porto Alegre, RS, Brasil, 2011.

[6] KUBOKI, T., AKIYAMA, M., NEISHI, Y., et al., "Effect of final drawing with light reduction on the leveling of residual stress distribuition in cold bar drawing”, Proceedings of the Institution of Mechanical Engineers, v. 214, n.11, pp. 1389 - 1400, Nov. 2000.

[7] NUNES, R. M., Análise de Tensões Residuais de Aços AISI 148 Produzidos por Trefilação Combinada Visando Minimizar Distorções Pós-Processamento, PPGE3M/UFRGS, Porto Alegre, RS, Brasil, 2008. 
[8] NUNES, R. M., Estudo de Distorção de Barras Cilíndricas de Aço ABNT 1045 em uma Rota de Fabricação Envolvendo Trefilação Combinada e Tempera por Indução, PPGE3M/UFRGS, Porto Alegre, RS, Brasil, 2012.

[9] ÖVERSTAM, H., "The influence of bearing geometry on the residual stress state in cold drawn wire, analysed by the FEM”, Journal of Materials Processing Technology, v. 171, n. 3, pp. 446-450, Fev. 2006.

[10] SOARES, C. A. T., Simulação Numérica das Tensões Residuais no Processo de Trefilação Considerando os Efeitos de Anisotropia e Distorções, PPGE3M/UFRGS, Porto Alegre, RS, Brasil, 2012.

[11] ASAKAWA, M., SASAKI, W., SHISHIDO, S., "Effect of die approach geometry and bearing length on residual stress after bar drawing”, Wire Journal International, v.35, n. 10, Out. 2002.

[12] RIPOLL, M. R., WEYGAND, S. M., RIEDEL, H. "Reduction of tensile residual stresses during the drawing process of tungsten wires”, Materials Science and Engineering: A, v.527, n.13-14, pp. 3064-3072, Mai. 2010.

[13] TEKKAYA, A. E., “State-of-the-art of simulation of sheet metal forming”, Journal of Materials Processing Technology, v. 103, n. 1, pp. 14-22, Jun. 2000.

[14] WOO, D. M., “On the complete solution of the deep-drawing problem”, International Journal of Mechanical Sciences, v. 10, n. 2, pp. 83-94, Fev. 1968.

[15] KUBOKI, T., AKYIAMA, M., NEISHI, Y., et al., "Effect of die geometry on residual stress level present after bar drawing”, Ironmaking \& Steelmaking, v. 28, n. 1, pp. 65-71, 2001.

[16] LANGE, K., Handbook of Metal Forming, 1 ed., Dearborn, Society of Manufacturing En-gineers, 1995.

[17] DAGNESE, J., Análise do Comportamento Elasto-plástico de um Aço AISI 1045 em Baixas Deformações, PPGE3M/UFRGS, Porto Alegre, RS, Brasil, 2012.

[18] ALTAN, T., OH, S., GEGEL, H. L., Conformação dos Metais: Fundamentos e Aplicações, 1 ed., São Paulo, EDUSP, 1999.

[19] JOHNSON, R. W., ROWE, G. W., "Redundant work in drawing cylindrical stock”, Journal of the Institute of Metals, v. 96, pp. 97-105, 1968.

[20] SCHAEFFER, L., Conformação Mecânica, 3 ed., Porto Alegre, Imprensa Livre, 2009. 